\title{
Functional Outcomes After Treatment of Posterior Inferior Cerebellar Artery Aneurysms
}

Mostafa Fatehi ${ }^{1}$, Michael A. Rizzuto ${ }^{1}$, Swetha Prakash ${ }^{1}$, Charles Haw ${ }^{1}$, Peter A. Gooderham ${ }^{1}$, Gary J. Redekop $^{1}$

1. Division of Neurosurgery, Vancouver General Hospital, Vancouver, CAN

Corresponding author: Mostafa Fatehi, fatehi@alumni.ubc.ca

\section{Abstract \\ Objective}

Aneurysms of the posterior inferior cerebellar artery (PICA) are a rare cause of subarachnoid hemorrhage. Treatment for this type of aneurysm may be microsurgical clipping or endovascular. This decision is based on patient characteristics, aneurysm location and dimensions, along with surgeon and institutional experience. In this study we aim to assess the outcomes of surgical and endovascular treatment of PICA aneurysms.

\section{Methods}

We retrospectively reviewed the charts of 52 patients who were admitted to Vancouver General Hospital for ruptured or symptomatic PICA aneurysms between 2005 and 2015. Modified Rankin scores were assigned at the time of discharge and at two subsequent follow-up time points. The mean short-term follow-up period post-operatively was 11.1 months and the mean long-term follow-up period was 19.3 months. Clinical and radiological characteristics were collected for all patients.

\section{Results}

Of the 52 patients, two died prior to obtaining treatment. Of the 50 patients who were treated for their PICA aneurysm, 39 presented with subarachnoid hemorrhage while 11 had symptomatic unruptured PICA aneurysms. Overall, 11 patients had endovascular treatment (coil embolization) while 39 patients underwent microsurgical clipping/trapping of the aneurysm. At the time of hospital discharge, patients in the microsurgical group trended towards a better the modified Rankin Scale score (2.3) compared to the endovascular group, though this did not reach significance (3.0) $(\mathrm{p}=0.20)$. The long-term score in the endovascular group (1.6) was also comparable to the microsurgical group (1.9) $(\mathrm{p}=0.55)$.

\section{Conclusion}

While the early outcomes in patients treated endovascularly appear better, there is no statistically

Review began 09/14/2020 Review ended 11/27/2020 Published 11/28/2020

\section{() Copyright 2020}

Fatehi et al. This is an open access article distributed under the terms of the Creative Commons Attribution License CC-BY 4.0., which permits unrestricted use, distribution, and reproduction in any medium, provided the original author and source are credited. significant difference in outcomes between the microsurgical and endovascular treatment groups at shortand long-term follow-up.

\section{Categories: Neurosurgery}

Keywords: endovascular aneurysm repair, microsurgical aneurysm clipping, posterior inferior cerebellar artery, subarachnoid hemorrhage

\section{Introduction}

Aneurysms of the posterior inferior cerebellar artery (PICA) are a rare cause of subarachnoid hemorrhage and account for $0.5-3 \%$ of all intracranial aneurysms [1-2]. Treatment for ruptured and symptomatic PICA aneurysms may be microsurgical clipping [3-5] or endovascular (including coil embolization, flow diverting stent reconstruction, and occasionally proximal occlusion) [6-8]. Treatment choice is affected by patient characteristics, aneurysm location and dimensions, as well as surgical and institutional experience. Furthermore, treatment is challenged by a high proportion of fusiform aneurysms, the tortuosity of the PICA, and relative proximity to lower cranial nerves in the posterior fossa [9]. Consequently, it was previously reported that patients with ruptured PICA aneurysms had a relatively poor clinical outcome [1012].

Due to their rarity, there is a dearth of high-quality outcome studies for PICA aneurysms. Two large studies that attempted to assess outcomes for ruptured PICA aneurysms are the Barrow Ruptured Aneurysm Trial (BRAT) [12] and International Subarachnoid Aneurysm Trial (ISAT) [13]. These represent two of the largest aneurysm trials published. Nevertheless, conclusions from these studies guiding the treatment of PICA aneurysms are limited by the small sample size of the PICA cohort. To date, several case series have reported variable treatment outcomes with either treatment modality. However, there is a scarcity of data regarding 
More recently, two groups have published retrospective analyses of their patients with PICA aneurysms. The first, by Bohnstedt et al. [10], reported the early post-treatment outcomes for PICA or vertebral artery aneurysms and found that the most significant predictors of outcome were age and presenting Hunt-Hess (HH) score and not aneurysm location or management modality. However, they also noted significantly higher complications after clipping compared to endovascular treatment. A multicenter registry review from Switzerland reported functional outcomes for 62 patients [11]. Two-thirds of these patients underwent endovascular treatment and the authors found generally good outcomes at discharge or one-year follow-up. However, the effect of treatment choice was not reported in this study.

In this study, we present the clinical characteristics and functional outcomes of patients undergoing clipping or coiling for ruptured and unruptured PICA aneurysms at Vancouver General Hospital (VGH) from 2005 to 2015 with the aim of determining if outcomes between treatment modalities are equivalent.

\section{Materials And Methods}

This study is a single-center retrospective review of a prospectively maintained database of patients admitted to VGH with unruptured or ruptured PICA aneurysms. Due to advances in endovascular management in the past two decades, data collection was limited to patients admitted between 2005 and 2015. From the VGH Neurosurgical Database, 52 patients with PICA aneurysms were identified. Variables collected include patient demographics, clinical presentation, Hunt-Hess scores, treatment complications, radiological characteristics, and hospital length of stay (LOS). Long-term outcomes were obtained by reviewing the charts of treating neurosurgeons. A score on the modified Rankin scale (mRS) was assigned for each patient at the time of hospital discharge and at follow-up appointments with their treating surgeon. Post-operative follow-up visit timelines vary in each surgeon's practice. The "short-term follow-up" timepoint in this study was the first post-operative appointment where mRS was recorded while the "longterm follow-up" timepoint was approximately one year later. A simple non-parametric T-test was used to compare mean mRS scores and clinical characteristics over time, between treatment modalities, and between ruptured and unruptured presentations (Prism 7, Graphpad Software, La Jolla, CA, USA, ). This study was approved by the University of British Columbia Clinical Research Ethics Board, approval \#H1702147.

A total of 52 patients were admitted with unruptured or ruptured PICA aneurysms during the study period. Of the 52 patients, two patients with subarachnoid hemorrhage (SAH) died prior to the treatment of their PICA aneurysm. There was a strong predilection toward females presenting with ruptured aneurysms (Table 1). The mean age at presentation was 59.3 (SD 13.8) years old. Of the 50 treated patients, 39 presented with SAH and 11 were treated for symptomatic unruptured PICA aneurysms. Eleven patients had endovascular treatment while 39 patients underwent microsurgical clipping/trapping of the aneurysm. 


\section{Cureus}

\begin{tabular}{|c|c|c|c|c|}
\hline & Total & Ruptured & Unruptured & $\mathbf{p}$ \\
\hline Number Treated & 50 & 39 & 11 & \\
\hline \multicolumn{5}{|l|}{ Sex } \\
\hline Male & 16 & 15 & 1 & \\
\hline Female & 34 & 24 & 10 & $<0.05$ \\
\hline \multicolumn{5}{|l|}{ Age } \\
\hline Mean (SD) & $59.3(13.8)$ & $58.8(14.1)$ & $61.3(13.5)$ & 0.6 \\
\hline \multicolumn{5}{|l|}{ Location on PICA } \\
\hline Distal & 30 & 24 & 6 & \\
\hline Proximal & 20 & 15 & 5 & \\
\hline \multicolumn{5}{|c|}{ Hunt-Hess SAH Grade } \\
\hline 1 & 0 & 0 & - & \\
\hline II & 9 & 9 & - & \\
\hline III & 13 & 13 & - & \\
\hline IV & 11 & 11 & - & \\
\hline v & 2 & 2 & - & \\
\hline Not listed & 4 & 4 & - & \\
\hline
\end{tabular}

TABLE 1: Patient demographics and presentation characteristics

PICA: posterior inferior cerebellar artery, SAH: subarachnoid hemorrhage

\section{Results}

The majority of all treated aneurysm were occluded based on post-operative CT angiogram (Table 2). The mean hospital length of stay (LOS) was equivalent in the endovascular and microsurgical groups. When stratified by presentation, patients with ruptured aneurysms required more time in hospital (Table 3, Table 4). There was no difference between treatment modalities or treatment modality within all ruptured presentations when comparing the number of patients that were discharged home and those that were transferred to their local hospital or rehabilitation facility. However, a greater proportion of patients with unruptured aneurysms were discharged home directly after treatment as compared to those presenting with $\mathrm{SAH}$. Among all 50 patients, retreatment was only required for an unruptured aneurysm treated endovascularly. Functional outcomes characterized by modified Rankin scale scores at discharge were no different in the microsurgical group (2.3) compared to the endovascular group (3.0). This remained the case at one-year follow-up (mean=11.1 months) in the endovascular group (1.4) and microsurgical group (2.0). At long-term follow-up (mean=19.3 months), mRS remained equivalent between the endovascular (1.6) and microsurgical groups (1.9) (Table 2). When stratifying patients by ruptured or unruptured presentation (Table 3) or treatment modality within the ruptured group (Table 4), mRS scores at all time points remained equivalent. 


\section{Cureus}

\begin{tabular}{|c|c|c|c|}
\hline & Microsurgical & Endovascular & $\mathbf{p}$ \\
\hline Number Treated & 39 & 11 & \\
\hline \multicolumn{4}{|l|}{ Degree of Occlusion } \\
\hline Complete & 35 & 9 & \\
\hline Residual Neck & 2 & 1 & \\
\hline Residual Aneurysm & 2 & 1 & \\
\hline Retreatment & 0 & 1 & 0.34 \\
\hline \multicolumn{4}{|l|}{ Hospital Length of Stay } \\
\hline Mean days (SD) & $28.3(40.5)$ & $22.7(19.8)$ & 0.53 \\
\hline \multicolumn{4}{|l|}{ Modified Rankin Score } \\
\hline \multirow[t]{2}{*}{ Mean at discharge (SD) } & $2.3(1.2)$ & $3.0(1.3)$ & 0.20 \\
\hline & $\mathrm{n}=32$ & $\mathrm{n}=7$ & \\
\hline \multirow[t]{2}{*}{ Mean at short-term follow-up (SD) } & $2.0(1.2)$ & $1.4(0.8)$ & 0.12 \\
\hline & $n=31$ & $n=7$ & \\
\hline \multirow[t]{2}{*}{ Mean at long-term follow-up (SD) } & $1.9(1.0)$ & $1.6(0.9)$ & 0.55 \\
\hline & $n=19$ & $n=5$ & \\
\hline \multicolumn{4}{|l|}{ Disposition } \\
\hline Home & 23 & 5 & 0.54 \\
\hline Rehabilitation facility & 4 & 1 & \\
\hline Local hospital & 5 & 2 & \\
\hline Unknown & 6 & 2 & \\
\hline Mortality & 1 & 1 & \\
\hline
\end{tabular}

TABLE 2: Outcomes stratified by treatment modality 


\section{Cureus}

\begin{tabular}{|c|c|c|c|}
\hline & Ruptured & Unruptured & $\mathbf{p}$ \\
\hline Number Treated & 39 & 11 & \\
\hline \multicolumn{4}{|l|}{ Degree of Occlusion } \\
\hline Complete & 36 & 8 & \\
\hline Residual Neck & 1 & 2 & \\
\hline Residual Aneurysm & 2 & 1 & \\
\hline Retreatment & 0 & 1 & 0.34 \\
\hline \multicolumn{4}{|l|}{ Hospital Length of Stay } \\
\hline Mean days (SD) & $31.6(40.5)$ & $11.3(8.0)$ & $<0.05$ \\
\hline \multicolumn{4}{|l|}{ Modified Rankin Score } \\
\hline \multirow[t]{2}{*}{ Mean at discharge (SD) } & $2.5(1.3)$ & $2.1(1.2)$ & 0.40 \\
\hline & $\mathrm{n}=29$ & $n=10$ & \\
\hline \multirow[t]{2}{*}{ Mean at short-term follow-up (SD) } & $1.9(1.2)$ & $1.9(1.0)$ & 0.89 \\
\hline & $\mathrm{n}=29$ & $n=8$ & \\
\hline \multirow[t]{2}{*}{ Mean at long-term follow-up (SD) } & $1.9(1.0)$ & $1.4(1.1)$ & 0.37 \\
\hline & $\mathrm{n}=19$ & $n=5$ & \\
\hline \multicolumn{4}{|l|}{ Disposition } \\
\hline Home & 18 & 10 & $<0.05$ \\
\hline Rehabilitation facility & 6 & 0 & \\
\hline Local hospital & 5 & 1 & \\
\hline Unknown & 8 & 0 & \\
\hline Mortality & 2 & 0 & \\
\hline
\end{tabular}

\section{TABLE 3: Outcomes stratified by presentation}




\section{Cureus}

\begin{tabular}{|c|c|c|c|}
\hline & Ruptured Microsurgical & Ruptured Endovascular & $\mathbf{p}$ \\
\hline Number Treated & 31 & 8 & \\
\hline \multicolumn{4}{|l|}{ Degree of Occlusion } \\
\hline Complete & 29 & 7 & \\
\hline Residual Neck & 1 & 0 & \\
\hline Residual Aneurysm & 1 & 1 & \\
\hline Retreatment & 0 & 0 & \\
\hline \multicolumn{4}{|l|}{ Hospital Length of Stay } \\
\hline Mean days (SD) & $32.6(44.4)$ & $27.5(20.7)$ & 0.64 \\
\hline \multicolumn{4}{|l|}{ Modified Rankin Score } \\
\hline \multirow[t]{2}{*}{ Mean at discharge (SD) } & $2.4(1.2)$ & $3.0(1.4)$ & 0.40 \\
\hline & $n=24$ & $\mathrm{n}=5$ & \\
\hline \multirow{2}{*}{ Mean at short-term follow-up (SD) } & $2.0(1.2)$ & $1.6(0.9)$ & 0.42 \\
\hline & $\mathrm{n}=24$ & $n=5$ & \\
\hline \multirow[t]{2}{*}{ Mean at long-term follow-up (SD) } & $2.0(1.0)$ & $1.74(1.0)$ & 0.67 \\
\hline & $n=15$ & $n=4$ & \\
\hline \multicolumn{4}{|l|}{ Disposition } \\
\hline Home & 16 & 2 & 0.43 \\
\hline Rehabilitation facility & 4 & 1 & \\
\hline Local hospital & 4 & 2 & \\
\hline Unknown & 6 & 2 & \\
\hline Mortality & 1 & 1 & \\
\hline
\end{tabular}

TABLE 4: Outcomes in ruptured presentations stratified by treatment modality

Major complications included stroke, dysphagia, development of hydrocephalus, seizure and meningitis; complications classified as minor were urinary tract infections, hyponatremia, delirium and voice hoarseness (Table 5). Major complications were of similar incidence in ruptured presentations compared to unruptured presentations. Additionally, treatment modality did not impact development of major complications or minor complications. Minor complications were more common in patients with ruptured presentations as compared to unruptured presentations. 


\section{Cureus}

\begin{tabular}{|c|c|c|c|c|c|}
\hline \multirow{2}{*}{ Complication } & \multicolumn{2}{|c|}{ Microsurgical (n=39) } & \multicolumn{2}{|l|}{ Endovascular $(n=11)$} & \multirow{2}{*}{ p } \\
\hline & Ruptured & Unruptured & Ruptured & Unruptured & \\
\hline \multicolumn{6}{|l|}{ Major } \\
\hline Death & 1 & 0 & 1 & 0 & \\
\hline Stroke & 2 & 1 & 1 & 0 & \\
\hline Cerebral & 1 & 1 & 0 & 0 & \\
\hline Cerebellar & 1 & 0 & 1 & 0 & \\
\hline Dysphagia & 2 & 0 & 0 & 1 & \\
\hline Hydrocephalus & 3 & 0 & 2 & 0 & \\
\hline Seizure & 1 & 0 & 0 & 0 & \\
\hline Meningitis & 1 & 0 & 0 & 0 & \\
\hline Microsurgical Subtotal & 11 & & Endovascular Subtotal & 5 & 0.45 \\
\hline Ruptured Subtotal & 14 & & Unruptured Subtotal & 2 & 0.25 \\
\hline \multicolumn{6}{|l|}{ Minor } \\
\hline Urinary Tract Infection & 3 & 0 & 0 & 0 & \\
\hline Voice Hoarseness & 2 & 0 & 0 & 0 & \\
\hline Pneumonia & 3 & 0 & 2 & 0 & \\
\hline Hyponatremia & 3 & 0 & 1 & 0 & \\
\hline Delirium & 0 & 0 & 2 & 0 & \\
\hline Microsurgical Subtotal & 11 & & Endovascular Subtotal & 5 & 0.34 \\
\hline Ruptured Subtotal & 16 & & Unruptured Subtotal & 0 & $<0.05$ \\
\hline
\end{tabular}

TABLE 5: Complications by treatment modality and rupture status

\section{Discussion}

Aneurysms of the posterior inferior cerebellar artery are a relatively uncommon cause of subarachnoid hemorrhage. Their treatment is complicated by a multitude of factors including but not limited to, close proximity to cranial nerves and the brainstem as well as higher rates of hemorrhage. The literature suggests that PICA aneurysms have worse functional outcomes as compared to other cerebral aneurysms [4,12-14]. However, there is limited existing literature regarding differences in outcomes when comparing treatment modality for PICA aneurysms.

With 31 ruptured PICA aneurysms in the study, the multicenter ISAT trial did not include a sufficient number of PICA aneurysms to yield a meaningful conclusion on their management. Patients were assessed at two months and one year post-operatively, but it is unclear how many of the 31 PICA patients were included at each timepoint [6].

The BRAT trial included 21 ruptured PICA aneurysms and found that despite presenting with similar HuntHess (HH) [15] scores to other posterior circulation aneurysms, PICA aneurysms were significantly associated with poorer outcomes (modified Rankin scale score $>2$ ). When compared to all non-PICA aneurysms at the time of discharge, one-year, three-year, and six-year follow-up, patients with PICA aneurysms consistently presented with statistically significant worse functional outcomes. At the six-year follow-up, $63 \%$ of PICA aneurysm patients had a poor outcome compared to $37 \%$ of non-PICA aneurysm patients [12]. The authors reported that this disparity may be attributed to the disproportionate proportion of PICA aneurysms randomized to the microsurgical arm (86\%). The BRAT trial concluded that much like treatment for other aneurysms, the choice of surgical vs. endovascular treatment for PICA aneurysms depends on patient characteristics and clinician expertise [14]. At one-year follow-up the BRAT demonstrated superiority of endovascular treatment for ruptured posterior circulation aneurysms with respect to functional outcomes [12]. However, in keeping with our findings the BRAT demonstrated no 
The present study is one of the largest single-institution reviews of treatment for PICA aneurysms to date. We present outcomes in a large series of patients that have undergone treatment for PICA aneurysms. Our institutional experience is fairly unique among North American centers in the preponderance of surgical management. Analysis of the present data suggests that while there are better short-term outcomes in patients treated endovascularly, there is no difference in mRS score between endovascular and microsurgical treatment of ruptured PICA aneurysms at follow-up. A favourable mRS has typically been defined as a score of 0 to 2 , and we see that in our cohort of patients surviving to follow-up there is a favourable outcome on average [10-12,16]. There was in fact a single patient with mRS of 4 at long-term follow-up in the microsurgically treated group. In comparing our findings to the literature, we find that microsurgical clipping techniques are in fact a safe and viable treatment choice for PICA aneurysms as demonstrated by a few retrospective series $[10,11,16]$.

When comparing ruptured PICA aneurysms to unruptured aneurysms we expectedly find that patients with SAH tend to have a longer hospital stay as they require extensive medical attention, long-term observation, and fewer patients are able to transition home at the time of discharge due to rehabilitation needs. Patients with SAH see a greater rate of minor complications compared to patients with unruptured aneurysms, which can likely be attributed to the secondary effects of SAH and the prolonged hospital stay required for these patients.

Due to the retrospective nature of the current study, a robust data set was not available with some data having not been collected and thus certain features could not be subjected to analysis. Additionally, analysis of our data is limited by the small population size. This potentially affects our ability to detect statistical differences between treatment groups. Given the sample size, the differences reported here may be influenced by patient comorbidities which potentially influenced treatment choice and may have predisposed certain patients to post-operative complications.

While PICA aneurysms are technically challenging to treat microsurgically given their proximity to lower cranial nerves and proximal segment of the PICA, it is often the preferred approach in aneurysms with challenging architecture. One might opt for a microsurgical approach in those aneurysms with a wide neck, fusiform appearance or those with a dissected or hypoplastic proximal segment or vertebral artery limiting endovascular access. Individual patient and aneurysm characteristics as well as surgeon experience may well have the greatest influence on outcomes when treating patients with PICA aneurysms.

\section{Conclusions}

Overall, functional outcomes after treatment of PICA aneurysms by endovascular or microsurgical means at Vancouver General Hospital over the 10-year period in question were equivalent at all follow-up time points. Patient disposition from hospital and average length of stay were also equivalent between the treatment modalities. As expected, patients presenting with ruptured PICA aneurysms were in hospital for significantly longer and discharged directly home to a lesser extent than those presenting in an unruptured fashion. Our study supports the notion that the treatment of PICA aneurysms should be decided based on patient characteristics, aneurysm architecture and surgeon experience as treatment modalities appear equivalent.

\section{Additional Information \\ Disclosures}

Human subjects: Consent was obtained by all participants in this study. University of British Columbia Clinical Research Ethics Board issued approval H17-02147. The UBC Clinical Research Ethics Board Chair or Associate Chair, has reviewed the above described research project, including associated documentation noted below, and finds the research project acceptable on ethical grounds for research involving human subjects and hereby grants approval. This approval applies to research ethics issues only. The approval does not obligate an institution or any of its departments to proceed with activation of the study. The Principal Investigator for the study is responsible for identifying and ensuring that resource impacts from this study on any institution are properly negotiated, and that other institutional policies are followed. The REB assumes that investigators and the coordinating office of all trials continuously review new information for findings that indicate a change should be made to the protocol, consent documents or conduct of the trial and that such changes will be brought to the attention of the REB in a timely manner. Animal subjects: All authors have confirmed that this study did not involve animal subjects or tissue. Conflicts of interest: In compliance with the ICMJE uniform disclosure form, all authors declare the following: Payment/services info: All authors have declared that no financial support was received from any organization for the submitted work. Financial relationships: All authors have declared that they have no financial relationships at present or within the previous three years with any organizations that might have an interest in the submitted work. Other relationships: All authors have declared that there are no other relationships or activities that could appear to have influenced the submitted work.

\section{References}


1. Lewis SB, Chang D, Peace DA, Lafrentz PJ, Day AL: Distal posterior inferior cerebellar artery aneurysms: clinical features and management. J Neurosurg. 2002, 97:756-766. 10.3171/jns.2002.97.4.0756

2. Tokimura H, Yamahata H, Kamezawa T, et al.: Clinical presentation and treatment of distal posterior inferior cerebellar artery aneurysm. Neurosurg Rev. 2011, 34:57-67. 10.1007/s10143-010-0296-Z

3. Drake C, Peerless S, Hernesniemi J: Surgery of the vertebrobasilar aneurysms: London, Ontario experience on 1767 patients. Springer, Vienna; 1996. 10.1007/978-3-7091-9409-6

4. Horowitz M, Kopitnik T, Landreneau F, Krummerman J, Batjer HH, Thomas G, Samson D: Posteroinferior cerebellar artery aneurysms: surgical results for 38 patients. Neurosurgery. 1998, 43:1026-1031. 10.1097/00006123-199811000-00012

5. Mascitelli JR, Yaeger K, Wei D, et al.: Multimodality treatment of posterior inferior cerebellar artery aneurysms. World Neurosurg. 2017, 106:493-503. 10.1016/j.wneu.2017.07.024

6. Bradac GB, Bergui M: Endovascular treatment of the posterior inferior cerebellar artery aneurysms . Neuroradiology. 2004, 46:1006-1011. 10.1007/s00234-004-1245-8

7. Chalouhi N, Jabbour P, Starke RM, et al.: Endovascular treatment of proximal and distal posterior inferior cerebellar artery aneurysms. J Neurosurg. 2013, 118:991-999. 10.3171/2012.12.JNS121240

8. Bhogal P, Chudyk J, Bleise C, Lylyk I, Henkes H, Lylyk P: The use of flow diverters to treat aneurysms of the posterior inferior cerebellar artery: report of three cases. Interv Neuroradiol. 2018, 24:489-498. 10.1177/1591019918774877

9. Lehto H, Harati A, Niemelä M, et al.: Distal posterior inferior cerebellar artery aneurysms: clinical features and outcome of 80 patients. World Neurosurg. 2014, 82:702-713. 10.1016/j.wneu.2014.06.012

10. Bohnstedt B, Ziemba-Davis M, Edwards G, et al.: Treatment and outcomes among 102 posterior inferior cerebellar artery aneurysms: a comparison of endovascular and microsurgical clip ligation. World Neurosurg. 2015, 83:784-793. 10.1016/j.wneu.2014.12.035

11. Starnoni D, Maduri R, Al Taha K, et al.: Ruptured PICA aneurysms: presentation and treatment outcomes compared to other posterior circulation aneurysms. A Swiss SOS study. Acta Neurochir (Wein). 2019, 161:1325-1334. 10.1007/s00701-019-03894-5

12. Spetzler RF, McDougall CG, Zabramski JM, et al.: The barrow ruptured aneurysm trial: 6-year results . J Neurosurg. 2015, 123:609-617. 10.3171/2014.9.JNS141749

13. Molyneux AJ, Kerr RSC, Yu L, Clarke M, Sneade M, Yarnold JA, Sandercock P: International Subarachnoid Aneurysm Trial (ISAT) of neurosurgical clipping versus endovascular coiling in 2143 patients with ruptured intracranial aneurysms: a randomised comparison of effects on survival, dependency, seizures, rebleeding, subgroups, and aneurysm occlusion. Lancet. 2005, 366:809-817. 10.1016/S0140-6736(05)67214-5

14. Spetzler RF, McDougall CG, Zabramski JM, et al.: Ten-year analysis of saccular aneurysms in the barrow ruptured aneurysm trial. J Neurosurg. 2020, 132:771-776. 10.3171/2018.8.JNS181846

15. Hunt WE, Hess RM: Surgical risk as related to time of intervention in the repair of intracranial aneurysms . J Neurosurg. 1968, 28:14-20. 10.3171/jns.1968.28.1.0014

16. Sejkorová A, Petr O, Mulino M, et al.: Management of posterior inferior cerebellar artery aneurysms: what factors play the most important role in outcome?. Acta Neurochir (Wein). 2017, 159:549-558. 10.1007/s00701-016-3058-z 